\title{
La aspirina sería igual de efectiva que la warfarina en la prevención de eventos tromboembólicos luego del reemplazo valvular aórtico con bioprótesis
}

Aspirin could be as effective as warfarin in the prevention of thromboembolic events after aortic valve replacement with a bioprosthesis

\section{Objetivos}

Comparar la eficacia y la seguridad del tratamiento con warfarina versus aspirina para prevenir eventos tromboembólicos durante los primeros tres meses posteriores a una cirugía de reemplazo valvular aórtico con prótesis biológica (RVAB).

\section{Diseño, lugar y pacientes}

Estudio unicéntrico, prospectivo, aleatorizado y abierto, realizado en The Heart Centre, Rigshospitalet, Copenhagen University Hospital, Dinamarca. Fueron invitados a participar mayores de 60 años en ritmo sinusal, derivados para RVAB con soporte, con o sin cirugía de revascularización miocárdica (CRM) concomitante.

\section{Intervenciones}

Fueron aleatorizados (1 a 1) el día previo a la cirugía a una de las siguientes ramas:

1) Enoxaparina $40 \mathrm{mg} /$ día subcutánea durante dos días o hasta lograr el rango de anticoagulación y warfarina iniciada el día posterior a la cirugía hasta alcanzar una Razón Internacional Normatizada (RIN) entre 2 y 3.

2) Enoxaparina subcutánea $40 \mathrm{mg} /$ día durante tres días y aspirina $150 \mathrm{mg} /$ día desde el primer día luego de la cirugía.

Los pacientes con CRM concomitante utilizaron además una dosis de $75 \mathrm{mg}$ diarios de aspirina.
Rafiq S y col. Thromb Res. 2017;150:104-10. El seguimiento fue durante tres meses, realizándose el análisis por intención de tratar.

\section{Medición de resultados principales}

El punto final primario fueron las complicaciones tromboembólicas (infarto de miocardio, accidente cerebrovascular, accidente isquémico transitorio, tromboembolismo pulmonar, trombosis venosa profunda, embolia periférica y trombo intracardíaco). Fueron puntos finales secundarios las complicaciones hemorrágicas como los sangrados mayores (riesgo de muerte $\mathrm{y} / \mathrm{o}$ necesidad de transfusión de más de una unidad de glóbulos rojos, hemorragia gastrointestinal, ACV hemorrágico) y menores; así como la mortalidad por cualquier causa.

\section{Resultados principales}

De los 370 pacientes que fueron aleatorizados entre 2005 y 2012, 328 completaron el seguimiento. Como lo muestra la tabla 1 , no se observaron diferencias estadísticamente significativas a los tres meses, en la incidencia de eventos tromboembólicos, sangrados totales, ni en la mortalidad total.

Los resultados no difirieron entre los pacientes sometidos a RVAB y los que además recibieron en forma combinada CRM. En el $79 \%$ del grupo warfarina se obtuvo información sobre los controles hematológicos. Sólo el $27,1 \%$ estuvo más del $75 \%$ del tiempo con un RIN en rango terapéutico y $12,7 \%$ tuvo al menos un valor por encima de 4,5 .

Tabla 1. comparación de la eficacia y la seguridad a los tres meses de dos alternativas terapéuticas en pacientes con reemplazo valvular aórtico con bioprótesis

\begin{tabular}{|c|c|c|c|}
\hline & Warfarina ( $n=167$ ) & Aspirina $(n=161)$ & $\mathbf{P}$ \\
\hline $\begin{array}{l}\text { Eventos tromboembólicos } \\
\text { Sangrados totales }\end{array}$ & $\begin{array}{l}11(6,6 \%) \\
9(5,4 \%)\end{array}$ & $\begin{array}{l}12(7,5 \%) \\
3(1,9 \%)\end{array}$ & $\begin{array}{r}10,83 \\
0,14\end{array}$ \\
\hline
\end{tabular}

El uso de warfarina resulto ser un predictor independiente de sangrado mayor (OR 5,18; IC95 \% 1,1 a 25,4; $\mathrm{p}=0.043$ ). No se encontraron variables predictoras de eventos tromboembólicos.

\section{Conclusiones}

La aspirina podría ser igualmente efectiva que la warfarina en la prevención de eventos tromboembólicos luego de un RVAB, pero con menor incidencia de sangrados mayores.

\section{Comentario}

A pesar del bajo riesgo de embolia a largo plazo, las bioprótesis valvulares parecieran ser moderadamente protrombóticas durante los primeros tres meses de colocadas, debido a la incompleta endotelización y el gradiente transvalvular que implican, ya que tienen una área efectiva menor que las nativas ${ }^{1}$. Estudios observacionales ${ }^{2,3}$ y pequeños estudios prospectivos ${ }^{4,5}$ no han podido responder cuál es el tratamiento antitrombótico óptimo tras el RVAB. Por ejemplo, en una cohorte de 25.656 pacientes la combinación aspirina-warfarina versus aspirina sola, redujo un $20 \%$ la mortalidad y un $48 \%$ la incidencia de embolia, pero casi triplicó el riesgo de sangrado. Sin embargo, no hubo diferencias entre warfarina sola y aspirina sola ${ }^{6}$. En pacientes con bajo riesgo trombótico las guías norteamericanas recomiendan con diferente fuerza la prescripción de aspirina (clase I) y de warfarina (clase lla); recomendando (clase I) warfarina en pacientes con alto riesgo tromboembólico (p. ej. fibrilación auricular, disfunción ventricular izquierda, tromboembolismo previo o estados hipercoagulable) ${ }^{7}$. Los europeos recomiendan el uso de aspirina durante tres meses como alternativa a la anticoagulación ${ }^{8,9}$. Los autores del estudio que hemos resumido plantean que la warfarina y la aspirina son similares en su eficacia para reducir la incidencia de eventos tromboembólicos luego de un RVAB, pero presentando la anticoagu- lación mayor incidencia de sangrado. Como en estudios previos ${ }^{4}$, el mantenimiento dentro del rango de anticoagulación óptimo resultó complejo, lo que podría haber generado un incremento de eventos embólicos o hemorrágicos en pacientes en quienes el RIN no haya estado en el rango óptimo.

Los nuevos anticoagulantes con menor variabilidad en su absorción, mayor estabilidad de sus niveles plasmáticos y de probado beneficio en el contexto de fibrilación auricular podrían resolver estas limitaciones pero aun no han sido probados en pacientes con RVAB. Vale destacar que en aquellas personas sometidas a reemplazo valvular mecánico, dabigatrán incrementó significativamente la incidencia de trombosis protésica y la de sangrado ${ }^{10}$.

\section{Conclusiones del comentador}

Luego de un RVAB la aspirina tendría similar efecto antitrombótico y sería más segura que la warfarina. Sin embargo, esto continúa siendo una hipótesis ya que este estudio carece de un poder estadístico suficiente como para responder este interrogante. Es por eso que en este contexto podemos pensar por ahora que la aspirina es una muy buena opción en pacientes de bajo riesgo tromboembólico, mientras que en los de alto riego la warfarina aún conserva su lugar. 
Ezequiel Berlante [ Servico de cardiología del Hospital Italiano de Buenos Aires. ezequiel.berlante@hospitalitaliano.org.ar ]

Berlante E. La aspirina sería igual de efectiva que la warfarina en la prevención de eventos tromboembólicos luego del reemplazo valvular aórtico con bioprótesis. Evid Act Pract Ambul. 2018;21(2): 48-49. Comentado de: Rafiq S y col. Antithrombotic therapy after bioprosthetic aortic valve implantation: Warfarin versus aspirin, a randomized controlled trial. Thromb Res. 2017;150:104-10. PMID: 27914653.

\section{Referencias}

1. Heras M y col. High risk of thromboemboli early after bioprosthetic cardiac valve replacement. J Am Coll Cardiol. 1995;25(5):1111-9. PMID: 7897124.

2. Aramendi JL y col. Prevention of thromboembolism with ticlopidine shortly after valve repair or replacement with a bioprosthesis. J Heart Valve Dis. 1998;7(6):610-4. PMID: 9870193

3. David T y col. Thromboembolism in Patients with Aortic Porcine Bioprostheses. Ann Thorac Surg. 1985;40(3):229-33. PMID: 4037914

4. ARAMENDI J y col. Triflusal versus oral anticoagulation for primary prevention of thromboembolism after bioprosthetic valve replacement (TRAC): rationale and design for a prospective, randomized, co-operative trial. Interact Cardiovasc Thorac Surg [Internet]. junio de 2003 [citado 13 de enero de 2018];2(2):170-4. Disponible en: http://www.ncbi.nlm. nih.gov/pubmed/17670019PMID: 17670019 .

nih.gov/pubmed/17670019PMID: 17670019 . 5. Colli A y col. Comparing warfarin to aspirin (WoA) after aortic valve
Hear Valve Dis. 2007;16(0966-8519 (Print)):667-71. PMID: 18095518.

6 . Brennan $\mathrm{J}$ y col. Early anticoagulation of bioprosthetic aortic valves in older patients: Results from the society of thoracic surgeons adult cardiac surgery national database. $\mathrm{J}$ Am Coll Cardiol. 2012;60(11):971-7. PMID: 22921973.

7. Bonow R y col. ACC/AHA 2006 Guidelines for the Management of Patients With Valvular Heart Disease. J Am Coll Cardiol [Internet]. 2006;48(3):e1-148. Disponible en: http:// linkinghub.elsevier.com/retrieve/pii/S0735109706012630PMID: 16875962.

8. Vahanian A y col. Guidelines on the management of valvular heart disease (version 2012). Eur Heart J. 2012;33(19):2451-96. PMID: 22922415.

9. Baumgartner $\mathrm{H}$ y col. $2017 \mathrm{ESC} / \mathrm{EACTS}$ Guidelines for the management of valvular heart disease. Eur Heart $\mathrm{J}$ [Internet]. 26 de agosto de 2017 [citado 13 de enero de 2018];38(36):2739-91. Disponible en: http://academic.oup.com/eurheartj/article/doi/10.1093/eurheartj/ehx391/4095039/2017-ESCEACTS-Guidelines-for-the-management-of 10. Eikelboom JW y col. Dabigatran versus warfarin in patients with mechanical heart valves. N Engl J Med [Internet]. 2013;369(13):1206-14. Disponible en: http://www.ncbi.nlm. nih.gov/pubmed/23991661PMID: 23991661 